\title{
Three models for resilient network design and a genetic algorithm to approach them
}

\author{
Jelena Sedlar ${ }^{1}$, Martina Milat ${ }^{1}$ \\ ${ }^{1}$ Faculty of civil engineering, architecture and geodesy, University of Split, \\ Matice hrvatske 15, HR-21000 Split, Croatia \\ (jelena.sedlar@gradst.hr, martina.milat@gradst.hr)
}

\begin{abstract}
This paper examines the types of directed networks with one source and one sink. The problem of resilient network design is studied with respect to such networks. The upper and the lower bound of the capacity are given for each edge in the network, while the cost of each edge is given as the function of edge capacity. Said problem of network design consists of selecting a subset of edges in the given network, which induces an optimal subnetwork to be resilient after disruptive event. The restoration behaviour of each edge in a network $N$ after the disruptive event is described by using a non-linear function that enables the modelling of three components affecting resilience: the remaining capacity of the edge after the disruption, the degree to which capability can be recovered and the recovery speed. Three different models for designing a resilient network are proposed and then formulated as problems of non-linear optimisation. A simple genetic algorithm using stochastic ranking, which can be used to approach all three proposed network design problems, is proposed. One numerical example is used to illustrate the proposed procedure and the effectiveness of the proposed algorithm.
\end{abstract}

Keywords: flow networks, design, resilience, genetic algorithm

MSC: $90 \mathrm{C} 27$

DOI: https://doi.org/10.5592/CO/CCD.2018.09 


\section{Introduction}

For the proper functioning of everyday life, we often rely on the regular functioning of many networks supporting our daily routine; from infrastructure networks, to traffic networks or information networks. Since disruptive events may occur which affect the functioning of those networks, there is an ever-increasing demand for ensuring their regular functioning by minimising the adverse effects of disruptive events. One possible solution is by increasing system redundancy, which is often the focus of reliability engineering [10]. This approach focusses on increasing the probability of a system to properly operate for a specified period of time. On the other hand, there is a resilience engineering approach which focuses more on improving the system's capability to recuperate from disruptive events in a sense that a desired level of performance is quickly recovered after the disruption [4]. The concept of resilience was first introduced in [8] which confirmed its significant role in maintaining the stability of ecological systems. Since then, this research topic has received increasing attention, and substantial effort has been dedicated to defining and to measuring system resilience [3]. In [2], an indicator has been developed to measure the component importance by quantifying its adverse impact on system resilience when the disruption affected that component.

There are many definitions of resilience [9] and all these definitions aim at better understanding of system resilience in different contexts. However, the methods for resilient infrastructure system design have not been so extensively studied. There are several related studies ( [5], [12]), however, since in reality many infrastructure systems exist in the form of networks, it is of the utmost interest to study the resilient network design methods ( $[1],[6],[7])$. One such study of resilient network design is [13] where the restoration behaviour is described by using a non-linear function that enables the modelling of more refined attributes of restoration at the component level. Three components influencing resilience are especially considered: the remaining capacity (absorptive ability), the degree to which capability can be recovered (restoration ability) and the recovery speed. The method for designing a resilient network is consequently formulated as the problem of non-linear optimisation.

A network is given as a directed graph with one source and one sink. When constructing a real-life infrastructure system, due to various technical reasons it is often impracticable to construct the connection between all nodes. Since the network considered here is a mathematical model of the infrastructure system intended for construction, the edges included in the network represent the connections which 
are technically feasible in the real-life. Generally, not every technically feasible connection is actually constructed in the infrastructure system, as it would often be too expensive and unnecessary. A satisfactory network can be obtained by constructing only some of the possible connections. Therefore, in designing the infrastructure system the engineer has to choose which feasible connections will be constructed. Mathematically, that means we have to choose a subset of edges in a given network which induces an optimal subnetwork with respect to the capacity, the cost and the resilience. In [13] said problem is approached by using a probabilistic solution discovery algorithm combined with stochastic ranking.

The proposed resilient network design model from [13] can be improved by allowing variable capacities of the edges and by introducing a variable cost of the edge construction defined as the function of the edge capacity. The aim of this paper is to implement these improvements in the resilient network design model and, consequently, to propose a simple genetic algorithm approach to the problem, since the probabilistic solution discovery algorithm proposed in [13] can hardly be extended to the model with variable edge capacities. Three different resilient network design models are proposed and then formulated as the problems of non-linear optimisation, and subsequently approached by a single algorithm. One numerical example is used to illustrate the proposed procedure and the effectiveness of the proposed method.

The present paper is structured as follows: the following section describes the preliminaries and introduces the basic notation. In Section 3, three different resilient network design models are proposed and then formulated as problems of non-linear optimisation. Section 4 presents a simple genetic algorithm combined with stochastic ranking which may be used to approach all three problems stated in Section 3. Finally, in Section 5, the algorithm presented in Section 4 is applied to one particular network $N$, and all three design problems are solved by using said algorithm. The effectiveness of the proposed method on the given example is verified by comparing the obtained results with the results obtained by exact calculation.

\section{Preliminaries}

Let $G=(V, E)$ be a directed graph on the set of vertices $V$ and the set of directed edges $E$. Directed edge $(v, w) \in E$ will often be denoted by abbreviation $v w$.

Definition 1 Flow network $N$ is defined by $N=(G, u, s, r)$, where $G=(V, E)$ is a directed graph, $u: E \rightarrow \mathbb{R}^{+}$is a non-negative edge capacity function, $s \in V$ is the 
source and $r \in V$ is the sink vertex.

The value $u(v w)$ of the edge capacity function $u$ in the network $N$ represents the capacity of the edge $v w \in E$ and will often be shortly denoted by $u_{v w}$.

Definition 2 Flow in the flow network $N=(G, u, s, r)$ is a non-negative function $\psi: E \rightarrow \mathbb{R}^{+}$such that $\psi(v w) \leq u(v w)$ for every $v w \in E$ and $\sum_{(w, v) \in E} \psi(w, v)=$ $\sum_{(v, z) \in E} \psi(v, z)$ for every vertex $v \in V \backslash\{s, r\}$. The value of the flow $\psi$ in the network $N$ is defined as $\psi(N)=\sum_{(s, v) \in E} \psi(s, v)$.

The standard problem in the flow networks theory is to find a flow in the network with the maximum possible value. Since the flow networks are often used to model various real-life problems, the designing or studying such networks involves the cost of building the network or similar costs. In addition, once it has been designed, the flow network exists in time, where a disruptive event can occur and diminish the edge capacity of several or all edges in the network. Since the original network is usually designed so that the maximum flow equals the amount of the actually required flow, it is of utmost interest to repair the network after the disruptive event as soon as possible. The value of the flow which is lost in a disruptive event $e^{d}$ is denoted by $\operatorname{Loss}\left(t_{d}\right)$, while the value of flow which can be recovered (of the flow which was lost) in the time $t$ after $t_{d}$ is denoted by Recovery $(t)$. The value of $\operatorname{Loss}\left(t_{d}\right)$ and Recovery $(t)$ is usually given by real-life circumstances and is therefore predefined in the analysed problem. Now we can define resilience function $R(t)$ of the network $N$ in the recovery time $t$ as

$$
R(t)=\frac{\operatorname{Recovery}(t)}{\operatorname{Loss}\left(t_{d}\right)} .
$$

Note that $R(t)$ is the percentage of the recovered flow in the recovery time $t$, hence $R(t) \leq 1$. Therefore, it is now possible to consider the maximum flow in the network, the cost of the network and the resilience of the network in the recovery time $t$.

One context in which we can consider the maximum flow, the cost and the resilience of a network is the context of network design. In [13] the authors studied the problem of designing a network with the lowest cost such that the designed network satisfies a given lower bound on resilience in the recovery time $t$. The concept of resilience is introduced by comparing two networks, the original network denoted by $N$ and the disrupted network after the recovery time $t$ which is denoted by $N^{*}(t)$. Both $N$ and $N^{*}(t)$ are based on the same directed graph $G$ where the vertices are 
denoted by $\{1, \ldots, n\}$ with vertex 1 being the source and vertex $n$ being the sink, they differ only in the edge capacity function.

The edge capacity function in the original undisrupted network $N$ is denoted by $u$, while the edge capacity function in the disrupted network $N^{*}(t)$ after the recovery time $t$ is denoted by $u^{*}(t)$. The values of the function $u$ for each edge of the network $N$ are predefined, while the value of the function $u^{*}(t)$ on the edge $i j \in E$ is denoted by $u_{i j}^{*}(t)$ and defined as the function

$$
u_{i j}^{*}(t)=u_{i j}\left(a_{i j}+\lambda_{i j}\left(1-a_{i j}\right)\left(1-e^{-b_{i j} t}\right)\right) .
$$

In this expression, $u_{i j}$ is the capacity of an edge $i j$ in the original network, $a_{i j}$ is the percentage of capacity $u_{i j}$ which remains operative in a disruptive event (hence, $1-a_{i j}$ is the percentage of capacity $u_{i j}$ lost in a disruptive event). Further, $\lambda_{i j}$ represents the percentage of the lost capacity $\left(1-a_{i j}\right) u_{i j}$ which can be recovered by repair. Finally, $b_{i j}$ denotes the speed of recovery of the edge $i j$. The values of $a_{i j}$, $b_{i j}$ and $\lambda_{i j}$ are predefined. Note that for $t=0$ we have $u_{i j}^{*}(t)=u_{i j} a_{i j}$, therefore the given function presupposes that $t=0$ is the time of the disruptive event, so $u_{i j}^{*}(t)$ denotes the capacity of the edge $i j$ in the recovery time $t$.

Terms $\psi(N)$ and $\psi\left(N^{*}(t)\right)$ denote the value of the maximum flow in networks $N$ and $N^{*}(t)$ respectively. The resilience function of the network $N$ is now defined as

$$
R(N, t)=\frac{\psi\left(N^{*}(t)\right)-\psi\left(N^{*}(0)\right)}{\psi(N)-\psi\left(N^{*}(0)\right)} .
$$

After the concept of resilience in a network is introduced, the problem of network design is postulated as follows. Network $N$ is a mathematical model of the infrastructure system an engineer has to construct, where connections between some nodes are technically feasible, while some other connections are technically impossible to construct. Therefore, the edges in the network $N$ represent the connections of the infrastructure system which are technically feasible. Since not all possible connections will be constructed, the engineer has to choose which connections to construct in order to obtain the optimal infrastructure system with respect to the capacity, the cost and the resilience. Mathematically, that means we have to find the subset of edges in $N$ which induces the optimal subnetwork of $N$. For a given network $N$, one such subset of edges is defined by the edge inclusion function $\delta: E \rightarrow\{0,1\}$ where

$$
\delta(i j)=\left\{\begin{array}{l}
0, \text { if the edge } i j \text { is not included in the constructed network } \\
1, \text { if the edge } i j \text { is included in the constructed network. }
\end{array}\right.
$$


We commonly use the abbreviated notation $\delta_{i j}$ instead of $\delta(i j)$. Consequently, the subnetwork of $N$, which is induced by the set of edges $E_{\delta}=\left\{i j \in E: \delta_{i j}=1\right\}$, is denoted by $N_{\delta}$.

Finally, the cost of the edge construction in the network $N$ is defined as the non-negative function $c: E \rightarrow \mathbb{R}^{+}$, where the cost $c(i j)$ of the edge $i j \in E$ is often denoted by abbreviation $c_{i j}$. The authors propose a network design model in which the subnetwork of the minimum cost is sought for, such that it has a minimum resilience required. In other words, they propose a network design model which is defined as the problem of nonlinear optimisation given by

$$
\text { minimize } f\left(N_{\delta}\right):=\sum_{i j \in E} \delta_{i j} c_{i j} \text { subject to } R\left(N_{\delta}, t\right) \geq \kappa .
$$

They also propose an improved probabilistic solution discovery algorithm for solving the proposed problem of nonlinear optimization. Finally, they provide two numerical examples on which their algorithm has been tested.

Since in real-life problems it is often possible to connect vertices $i$ and $j$ by the edge $i j$ of various capacities, it would be more realistic if in a proposed model one could choose which capacity $u_{i j}$ of the edge $i j$ to construct given the upper and lower capacity bound $\bar{u}_{i j}$ and $\underline{u}_{i j}$ respectively. In addition, the cost of construction of the edge $i j$ usually depends on the capacity, therefore a model would be more realistic if the cost were given as the function of edge capacity. Finally, although the authors of [13] mentioned that, in the problem of nonlinear optimisation an additional constraint can be added on the lower bound of the required value of the flow, they did not implement it in their example and therefore did not test the algorithm on said problem. The inherent problem of the model without the constraint on the value of the flow, but only with the minimized cost, is that we can obtain an optimal network which is only slightly cheaper than the rest, but the value of the maximum flow in it is significantly lower.

For instance, we may observe the network $N$ in Figure 1 (a), where vertex 1 is the source, while vertex 4 is the sink. The edge labels are the edge capacities and the cost of every edge equals 1 . As for the recovery parameters $a_{i j}, b_{i j}$ and $\lambda_{i j}$, suppose that $a_{i j}=0.3$ and $\lambda_{i j}=0.9$ for every edge $i j$, while $b_{i j}=0.7$ for $i j=13$, 34 and $b_{i j}=0.9$ for $i j=12,14,24$. In other words these recovery parameters mean that the edges 12,14 and 24 recover considerably faster than the edges 13 and 34 . Therefore, the both flow directions $1 \rightarrow 4$ and $1 \rightarrow 2 \rightarrow 4$ are substantially more resilient than the flow direction $1 \rightarrow 3 \rightarrow 4$. Let us consider two subnetworks of $N$, the first denoted by $N_{\delta}$ and shown in Figure 1 (b), the other is denoted by $N_{\delta^{\prime}}$ and 
shown in Figure 1 (c). If we apply the model from [13], where we minimise the cost given the constraint $R\left(N_{\delta}, 6\right)>0.8$, we will obtain network $N_{\delta}$ as optimal, since it is the cheapest with the cost equal to 1 and the required resilience $R\left(N_{\delta}, 6\right)=0.896$. However, the value of the maximum flow in that network equals $\psi\left(N_{\delta}\right)=1$. On the other hand, if we consider the network $N_{\delta^{\prime}}$, we note that at slightly greater cost 2 , we will obtain a similarly resilient network $\left(R\left(N_{\delta^{\prime}}, 6\right)=0.896\right)$, with a significantly greater value of the maximum flow $\left(\psi\left(N_{\delta^{\prime}}\right)=10\right)$. Therefore, from the economic perspective, the network $N_{\delta^{\prime}}$ is more frequently regarded as optimal.

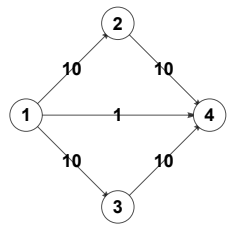

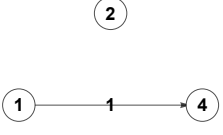

(3) a)

b)

Figure 1: a) The network $N$, b) the cheapest resilient subnetwork $N_{\delta}$, b) a slightly more expensive resilient subnetwork $N_{\delta^{\prime}}$ with a significantly greater value of the maximum flow.

The aim of this paper is to propose three models of resilient network design considering all the mentioned improvements and to propose a simple genetic algorithm for problem-solving. The proposed algorithm is tested on one numerical example for each of the models.

\section{Resilient network design models}

In order to describe the improved network design models, slightly different notation should be introduced. Let $G=(V, E)$ be a directed graph where vertices from $V$ are denoted by integers $1, \ldots, n$, with vertex 1 being the source and vertex $n$ being the sink. Let $\underline{u}: E \rightarrow \mathbb{R}^{+}$and $\bar{u}: E \rightarrow \mathbb{R}^{+}$be two non-negative functions such that $\underline{u}(i j) \leq \bar{u}(i j)$ for every $i j \in E$. The values $\underline{u}(i j)$ and $\bar{u}(i j)$ are often denoted by abbreviations $\underline{u}_{i j}$ and $\bar{u}_{i j}$ respectively. Let us denote by $N_{u}$ the network $(G, u, 1, n)$ where the edge capacity function $u$ satisfies the condition $\underline{u}_{i j} \leq u_{i j} \leq \bar{u}_{i j}$ for every $i j \in E$. If a disruptive event occurs in the network $N_{u}$ and diminishes the capacities of edges in $N_{u}$, then $N_{u}^{*}(t)$ denotes the disrupted network after the recovery time $t$. 
Therefore, networks $N_{u}$ and $N_{u}^{*}(t)$ differ only in the edge capacity function, where the capacity $u_{i j}^{*}(t)$ of the edge $i j$ in the network $N_{u}^{*}(t)$ is defined by

$$
u_{i j}^{*}(t)=u_{i j}\left(a_{i j}+\lambda_{i j}\left(1-a_{i j}\right)\left(1-e^{-b_{i j} t}\right)\right)
$$

as in the previous section, and the values of the recovery parameters $a_{i j}, b_{i j}$ and $\lambda_{i j}$ are predefined.

The value of the maximum flow in networks $N_{u}$ and $N_{u}^{*}(t)$ is denoted by $\psi\left(N_{u}\right)$ and $\psi\left(N_{u}^{*}(t)\right)$ respectively. Now, the resilience $R\left(N_{u}, t\right)$ of the network $N_{u}$ after the recovery time $t$ is also defined as in the previous section, i.e.

$$
R\left(N_{u}, t\right)=\frac{\psi\left(N_{u}^{*}(t)\right)-\psi\left(N_{u}^{*}(0)\right)}{\psi\left(N_{u}\right)-\psi\left(N_{u}^{*}(0)\right)} .
$$

Finally, the cost of the edge construction in the network $N_{u}$ is defined as the function $c: E \rightarrow \mathbb{R}^{+}$such that $c=h(u)$, where $h$ can be any function which gives the dependence of the edge cost and the edge capacity. The cost $c\left(N_{u}\right)$ of the network $N_{u}$ is then defined by

$$
c\left(N_{u}\right)=\sum_{i j \in E} c_{i j}
$$

We can now propose three models of resilient network design. In the first model the cost is minimised given the constraint on the value of the flow and the resilience, in the other model the value of the flow is maximised given the constraints on the resilience and the cost, and, finally, in the third model the resilience is maximised given the constraints on the flow value and the cost.

In order to formally describe the design models and define them as the problems of non-linear optimisation, we should primarily describe how the edge capacity function $u$ of the network $N_{u}$ can be written in the vector form. Let $m$ denote the number of edges in the network $N_{u}$. Since vertices in $N_{u}$ are denoted by integers $1, \ldots, n$, the edges $i j \in E$ can be lexicographically ordered. Therefore, we can define the edge capacity vector $\mathbf{u}$ by

$$
\mathbf{u}=\left(\ldots, u_{i j}, \ldots\right) \in \mathbb{R}^{m}
$$

where the order of capacities $u_{i j}$ in the $m$-tuple corresponds to the lexicographic order of the edges $i j \in E$. By analogy, we can define the vector $\mathbf{u}^{*}(t)$ for the capacity function $u^{*}(t)$ in the network $N_{u}^{*}(t)$. Since all networks $N_{u}$ are based on the same 
graph $G$ and differ only in the capacity function $u$, we can denote $\psi\left(N_{u}\right), R\left(N_{u}, t\right)$ and $c\left(N_{u}\right)$ by $\psi(\mathbf{u}), R(\mathbf{u}, t)$ and $c(\mathbf{u})$ and regard them as functions in the variable $\mathbf{u} \in \mathbb{R}^{m}$.

We will now consider the general non-linear programming problem formulated as

$$
\text { minimise } f(\mathbf{x}) \text { subject to } \mathbf{x} \in \mathcal{S} \cap \mathcal{F},
$$

where $f(\mathbf{x})$ is the objective function, $\mathcal{S}$ is the subset of $\mathbb{R}^{m}$ which consists of $\mathbf{x}=$ $\left(x_{1}, \ldots, x_{m}\right) \in \mathbb{R}^{m}$ satisfying the following constraints

$$
\underline{x}_{i} \leq x_{i} \leq \bar{x}_{i}
$$

and $\mathcal{F}$ is the subset of $\mathbb{R}^{m}$ consisting of $\mathbf{x} \in \mathbb{R}^{m}$ satisfying conditions $g_{j}(\mathbf{x}) \leq 0$ for $j=1, \ldots, p$, i.e.

$$
\mathcal{F}=\left\{\mathbf{x} \in \mathbb{R}^{m}: g_{1}(\mathbf{x}) \leq 0 \wedge \ldots \wedge g_{p}(\mathbf{x}) \leq 0\right\} .
$$

We will further define $g_{j}^{+}(\mathbf{x})=\max \left\{0, g_{j}(\mathbf{x})\right\}$ for every $j=1, \ldots, p$, therefore, by using a penalty function approach the constraint violations can be treated as a single penalty function

$$
\phi(\mathbf{x})=\sum_{j=1}^{p}\left(g_{j}^{+}(\mathbf{x})\right)^{2} .
$$

Now, a simple genetic algorithm can be developed in order to solve this nonlinear programming problem which uses stochastic ranking, as presented in the following section. We have to define an objective function $f(\mathbf{x})$ we minimise and the corresponding penalty function $\phi(\mathbf{x})$ for all three proposed models.

We can now propose three models of resilient network design.

Model 1. In this model, we minimise the cost subject to constraints on the value of flow and the resilience of the network. Therefore, the problem can be written as

$$
\begin{array}{ll}
\min & c(\mathbf{u}) \\
\text { s.t. } & \psi(\mathbf{u}) \geq \psi_{0} \text { and } \\
& R(\mathbf{u}, t) \geq R_{0}
\end{array}
$$


Therefore, in this model the objective function is $f(\mathbf{u})=c(\mathbf{u})$ subject to constraints $g_{1}(\mathbf{u})=\psi_{0}-\psi(\mathbf{u}) \leq 0$ and $g_{2}(\mathbf{u})=R_{0}-R(\mathbf{u}, t) \leq 0$. Now the penalty function $\phi(\mathbf{u})$ is defined by

$$
\phi(\mathbf{u})=\sum_{j=1}^{2}\left(g_{j}^{+}(\mathbf{u})\right)^{2}
$$

where $g_{1}^{+}(\mathbf{u})=\max \left\{\psi_{0}-\psi(\mathbf{u}), 0\right\}$ and $g_{2}^{+}(\mathbf{u})=\max \left\{R_{0}-R(\mathbf{u}, t), 0\right\}$.

Model 2. In this model, we maximise the value of the flow subject to constraints on the cost and the resilience of the network. Therefore, the problem can be written as

$$
\begin{array}{ll}
\max & \psi(\mathbf{u}) \\
\text { s.t. } & c(\mathbf{u}) \leq c_{0} \text { and } \\
& R(\mathbf{u}, t) \geq R_{0}
\end{array}
$$

In order to apply the same algorithm to all models, we will convert this problem to minimising, i.e. we define the objective function as $f(\mathbf{u})=-\psi(\mathbf{u})$ and then we minimise it subject to constraints $g_{1}(\mathbf{u})=c(\mathbf{u})-c_{0} \leq 0$ and $g_{2}(\mathbf{u})=R_{0}-R(\mathbf{u}, t) \leq 0$. Now the penalty function $\phi(\mathbf{u})$ is defined by

$$
\phi(\mathbf{u})=\sum_{j=1}^{2}\left(g_{j}^{+}(\mathbf{u})\right)^{2}
$$

where $g_{1}^{+}(\mathbf{u})=\max \left\{c(\mathbf{u})-c_{0}, 0\right\}$ and $g_{2}^{+}(\mathbf{u})=\max \left\{R_{0}-R(\mathbf{u}, t), 0\right\}$.

Model 3. In this model, we maximise the resilience of the network in the recovery time $t$ subject to constraints on the cost and the value of the flow in the network. Therefore, the problem can be written as

$$
\begin{array}{ll}
\max & R(\mathbf{u}, t) \\
\text { s.t. } & c(\mathbf{u}) \leq c_{0} \text { and } \\
& \psi(\mathbf{u}) \geq \psi_{0}
\end{array}
$$

Again, in order to apply the same algorithm to all models, we will convert this problem to minimising, i.e. we define objective function as $f(\mathbf{u})=-R(\mathbf{u}, t)$ and then minimize it subject to constraints $g_{1}(\mathbf{u})=c(\mathbf{u})-c_{0} \leq 0$ and $g_{2}(\mathbf{u})=\psi_{0}-\psi(\mathbf{u}) \leq 0$. Now the penalty function $\phi(\mathbf{u})$ is defined by

$$
\phi(\mathbf{u})=\sum_{j=1}^{2}\left(g_{j}^{+}(\mathbf{u})\right)^{2}
$$


where $g_{1}^{+}(\mathbf{u})=\max \left\{c(\mathbf{u})-c_{0}, 0\right\}$ and $g_{2}^{+}(\mathbf{u})=\max \left\{\psi_{0}-\psi(\mathbf{u}), 0\right\}$.

After proposing three different models of resilient network design, defined as the problems of non-linear optimisation, we will propose an efficient algorithm for solving said problems. Therefore, in the next section we will describe a simple genetic algorithm for solving these nonlinear optimization problems and then apply it to the numerical example in the following section.

\section{Algorithm for solving resilient network design prob- lem}

We want to propose a simple genetic algorithm for solving the non-linear optimisation problems to which a resilient network design models reduce. Since all considered networks are based on the same directed graph $G$ and differ only in the edge capacity function $u$, we will represent the network $N_{u}$ by its edge capacity vector $\mathbf{u}$ throughout the rest of the paper. In the first step of the algorithm, a population of $\lambda$ networks $\mathcal{G}^{(1)}=\left\{\mathbf{u}_{1,1}, \ldots, \mathbf{u}_{\lambda, 1}\right\}$ is generated at random, so that the constraint $\underline{u}_{i j} \leq u_{i j} \leq \bar{u}_{i j}$ is satisfied for every edge $i j$ in each individual $\mathbf{u}_{k, 1}$. This population represents the first generation of individuals. In addition, a generation counter $g$ is set to 0, i.e. $g \leftarrow 0$. Until the stopping criterion has been satisfied, the following steps are repeated. Firstly, the generation counter is set to $g+1$, i.e. $g \leftarrow g+1$. The individuals from population $\mathcal{G}^{(g)}=\left\{\mathbf{u}_{1, g}, \ldots, \mathbf{u}_{\lambda, g}\right\}$ are ranked using the procedure of stochastic ranking (to be subsequently explained) in order to obtain a new order of those individuals where $I_{j}$ is the ranking of $j$-th individual $\mathbf{u}_{j, g}$ from generation $g$. The $\mu$ best ranked individuals $\mathbf{u}_{j, g}$ are denoted by $\mathbf{u}_{I_{j}, g}^{\prime}$ and selected to be the set of genitors $\mathcal{P}^{(g)}=\left\{\mathbf{u}_{1, g}^{\prime}, \ldots, \mathbf{u}_{\mu, g}^{\prime}\right\}$ of the next generation. The next generation consists of all genitors from the previous generation (i.e. $\mathcal{P}^{(g)} \subseteq \mathcal{G}^{(g+1)}$ ) and all children generated by all parents from $\mathcal{P}^{(g)}$.

In order for $\mathcal{G}^{(g+1)}$ to have $\lambda$ individuals, besides $\mu$ parents from $\mathcal{G}^{(g)}$ which are included in $\mathcal{G}^{(g+1)}, \lambda-\mu$ children have to be generated. Therefore, for $k=1, \ldots, \lambda-$ $\mu$, the child $\mathbf{u}_{k, g+1}$ is generated from the genitor $\mathbf{u}_{i, g}^{\prime}$ where $i=(k-1) \bmod \mu+1$. In order to explain how the child $\mathbf{u}_{k, g+1}$ is generated from the genitor $\mathbf{u}_{i, g}^{\prime}$, let us recall that $\mathbf{u}_{i, g}^{\prime} \in \mathbb{R}^{m}$ where $m$ is the number of edges in the network. Each of the $m$ coordinates in $\mathbf{u}_{i, g}^{\prime}$ represents the capacity of the corresponding edge $i j$ and can be regarded as a gene. Mutation can occur in every gene in the sense that the capacity of that edge can be changed which results in a new 'mutated' network. 
If the number of mutations is lower and the size of mutation (i.e. the amount of capacity change in a gene which represents a certain edge $i j$ ) is smaller, the mutated child $\mathbf{u}_{k, g+1}$ is more similar to its parent $\mathbf{u}_{i, g}^{\prime}$. Therefore, the child $\mathbf{u}_{k, g+1}$ of the genitor $\mathbf{u}_{i, g}^{\prime}$ is created so that a random number $n_{m u t}$ of mutations is generated with half-normal distribution along the integers from $[1,2 n / 3]$. The $n_{m u t}$ coordinates in $\mathbf{u}_{i, g}^{\prime}$ where a mutation occurs are selected at random. Now, if a mutation is to occur in the coordinate corresponding to the edge $i j$ in $\mathbf{u}_{i, g}^{\prime}$, then the size $\delta_{i j}$ of the mutation is selected as a random number with normal distribution along the interval $\left[-\left(\bar{u}_{i j}-\underline{u}_{i j}\right), \bar{u}_{i j}-\underline{u}_{i j}\right]$. Assuming that $u_{i j}^{\prime}$ denotes the value of the $i j$ coordinate in $\mathbf{u}_{i, g}^{\prime}$, then if $u_{i j}^{\prime}+\delta_{i j}$ doesn't satisfy the constraint $\underline{u}_{i j} \leq u_{i j}^{\prime}+\delta_{i j} \leq \bar{u}_{i j}$, the process is repeated until it does. Furthermore, the genitors from $\mathcal{P}^{(g)}$ are included in $\mathcal{G}^{(g+1)}$ by setting $\mathbf{u}_{k, g+1}=\mathbf{u}_{k-(\lambda-\mu), g}^{\prime}$ for $k=\lambda-\mu+1, \ldots, \lambda$. The entire process is repeated with $\mathcal{G}^{(g+1)}$ and so on until the stopping criterion has been satisfied. The pseudocode of this algorithm is shown in Figure 2, while the process of offspring generation is given as the separate procedure whose pseudocode is shown in Figure 3.

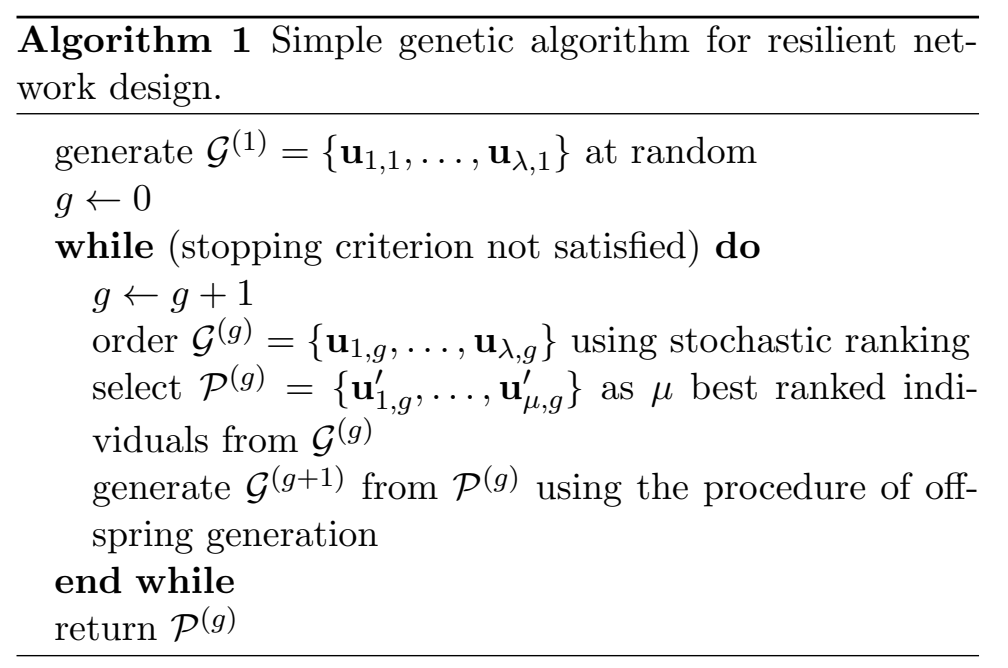

Figure 2: A simple genetic algorithm for resilient network design.

In each generation of $\lambda$ individuals, only $\mu$ best ranked individuals are selected to become parents of the next generation. The ranking of the $\lambda$ individuals is done by the stochastic ranking procedure [11] which is a bubble-sort-like procedure where a 


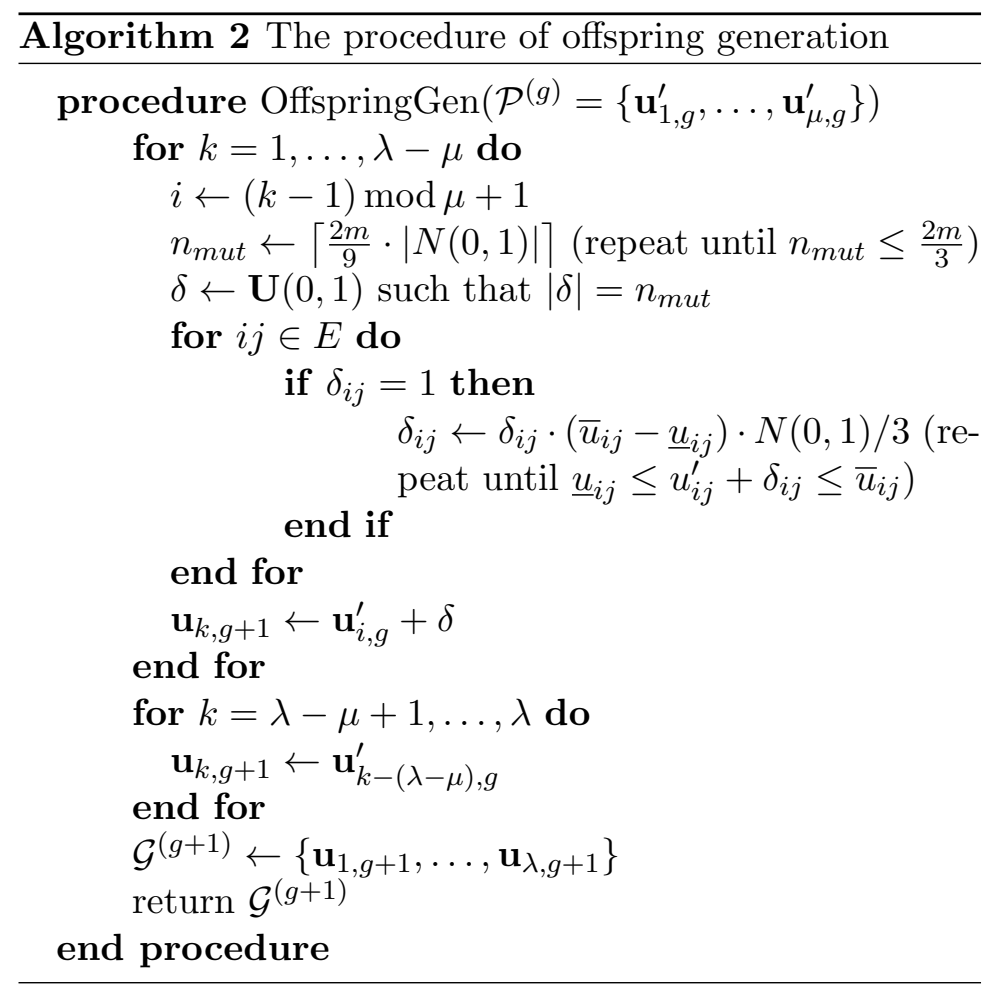

Figure 3: The procedure of offspring generation, $N(0,1)$ is a random number generated with normal distribution, while $\mathbf{U}(0,1)$ is randomly generated vector from $\{0,1\}^{m}$ with uniform distribution.

probability $P_{f}$ of using only the objective function for comparing individuals in the infeasible region of the search space is introduced. Namely, when two individuals are compared in order to determine which one is more suitable and should therefore be better ranked, if both individuals are feasible (the penalty function $\phi$ equals zero for both individuals), then the probability to rank those two individuals according to the value of the objective function is 1 , otherwise that probability is $P_{f}$. The procedure provides a convenient way of balancing the dominance in a ranked set. The pseudocode of the procedure of stochastic ranking is shown in Figure 4. Since we, eventually, want to obtain only feasible solutions, the probability $P_{f}$ should be set to be less than 0.5 so that there is a pressure against infeasible solutions. 
The value of $P_{f}$ is usually set to be 0.45 as the previous studies have shown that $P_{f}=0.45$ is often sufficient.

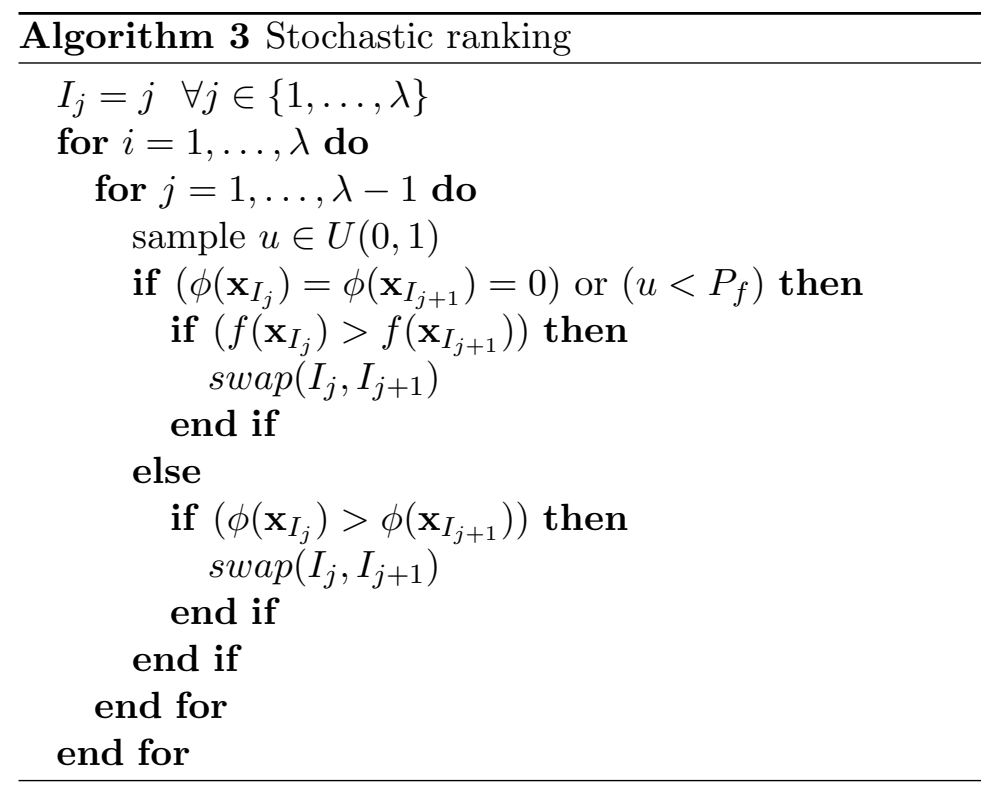

Figure 4: Stochastic ranking procedure, $P_{f}=0.45$.

\section{Numerical example}

Finally, we applied the proposed resilient network design models on graph $G$ and used the proposed genetic algorithms to solve those models. Firstly, we define the directed graph $G$ to which we will apply our models. Let $G=(V, E)$ be a graph with 6 vertices and 9 directed edges as shown in Figure 5.

Vertex 1 is the source and vertex 6 is the sink. In addition, let

$$
\overline{\mathbf{u}}=(4,3,2,2,2,3,1,3,4)
$$

be the vector whose coordinates $\bar{u}_{i j}$ are the upper bounds on the edge capacities $u_{i j}$. The coordinates $\bar{u}_{i j}$ in the vector $\overline{\mathbf{u}}$ are ordered according to the lexicographic order of the edges, i.e. $\overline{\mathbf{u}}=\left(\bar{u}_{12}, \bar{u}_{13}, \bar{u}_{23}, \bar{u}_{24}, \bar{u}_{34}, \bar{u}_{35}, \bar{u}_{45}, \bar{u}_{46}, \bar{u}_{56}\right)$. The same edge order will be used throughout this section. The lower bounds on edge capacities 


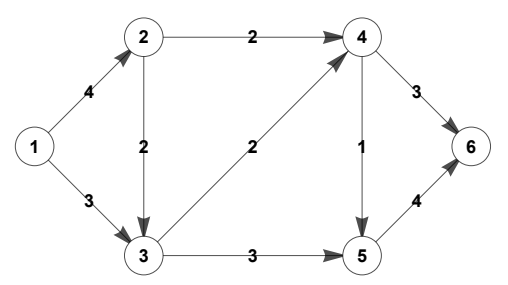

Figure 5: The directed graph $G$ which to which the proposed network design models will be applied.

\begin{tabular}{|c||c|c|c|c|c|c|c|c|c|}
\hline$i j$ & 12 & 13 & 23 & 24 & 34 & 35 & 45 & 46 & 56 \\
\hline \hline$a_{i j}$ & 0.37 & 0.21 & 0.47 & 0.28 & 0.43 & 0.25 & 0.33 & 0.44 & 0.31 \\
\hline$b_{i j}$ & 0.92 & 0.78 & 0.88 & 0.81 & 0.87 & 0.81 & 0.79 & 0.91 & 0.82 \\
\hline$\lambda_{i j}$ & 0.9 & 0.9 & 0.9 & 0.9 & 0.9 & 0.9 & 0.9 & 0.9 & 0.9 \\
\hline
\end{tabular}

Table 1: Recovery parameters $a_{i j}, b_{i j}$ and $\lambda_{i j}$ of the edge $i j$.

are defined by $\underline{u}_{i j}=0$ for every $i j \in E$, i.e. $\underline{\mathbf{u}}=\mathbf{0}$. In order to verify the optimal solution obtained by the algorithm, we want to be able to calculate the optimum exactly, so will allow only integer capacity values between $\underline{u}_{i j}$ and $\bar{u}_{i j}$. Therefore, this example has 86400 different possible networks in total. The cost of an individual edge of capacity $u_{i j}$ is defined as $c\left(u_{i j}\right)=0.3+u_{i j}$, while the cost of the network $N_{u}$ represented by the vector $\mathbf{u}$ is consequently defined as

$$
c(\mathbf{u})=\sum_{i j \in E, u_{i j} \neq 0} c\left(u_{i j}\right) .
$$

Finally, the recovery parameters $a_{i j}, b_{i j}$ and $\lambda_{i j}$ of the edge $i j$ are presented in the Table 1. We want to design an optimal network according to each of the proposed three resilient network design models by using the proposed genetic algorithm.

Model 1. Let us recall that in this model the cost is minimised subject to constraints on the value of flow and the resilience of the network. Therefore, the problem can be written as

$$
\begin{array}{ll}
\min & c(\mathbf{u}) \\
\text { s.t. } & \psi(\mathbf{u}) \geq \psi_{0} \text { and } \\
& R(\mathbf{u}, t) \geq R_{0}
\end{array}
$$




\begin{tabular}{|c|c|c|c|c|c|}
\hline$t$ & $n_{\text {gen }}$ & $\mathbf{u}_{\text {opt }}$ & $\psi\left(\mathbf{u}_{\text {opt }}\right)$ & $c\left(\mathbf{u}_{\text {opt }}\right)$ & $R\left(\mathbf{u}_{\text {opt }}, t\right)$ \\
\hline \hline 1 & 33 & $(2,3,0,2,2,1,0,3,1)$ & 4 & 16.1 & 0.590 \\
\hline 2 & 51 & $(2,3,0,2,2,2,0,3,1)$ & 4 & 17.1 & 0.786 \\
\hline 3 & 49 & $(2,3,0,2,1,2,0,2,2)$ & 4 & 16.1 & 0.841 \\
\hline 4 & 31 & $(2,3,0,2,1,2,0,2,2)$ & 4 & 16.1 & 0.879 \\
\hline 5 & 44 & $(2,3,0,2,2,1,0,3,1)$ & 4 & 16.1 & 0.904 \\
\hline 6 & 50 & $(2,3,0,2,1,2,0,2,2)$ & 4 & 16.1 & 0.902 \\
\hline
\end{tabular}

Table 2: The computational results of Model 1 by using the proposed genetic algorithm.

We set that $\psi_{0}=4$ and $R_{0}$ equals $0.58,0.779,0.838,0.878,0.892,0.9$ for $t=1, \ldots, 6$ respectively. By using these set values, we apply the proposed genetic algorithm to the problem of non-linear optimisation. When applying the genetic algorithm, the concrete values of the algorithm parameters, namely the size $\lambda$ of each generation, the number $\mu$ of genitors in every generation and the stopping criterion, must be selected. In all these models, the same values and stopping criterion are used. Firstly, the number of individuals in each generation is set to $\lambda=400$, the number of the most suitable individuals to become genitors of the next generation is set to $\mu=$ 50 , the stopping criterion is set in a way that the number of generations is at most $g_{\max }=200$ or that the set of different genitors in $\mathcal{P}^{(g)}$ is the same in 10 consecutive generations. The results obtained by using the proposed genetic algorithm are shown in Table 2 , where $n_{\text {gen }}$ denotes the number of generations produced by the algorithm before the stopping criterion was satisfied. Since $n_{\text {gen }} \leq 200=g_{\max }$ for every $t$, it follows that for each $t$ the execution of the algorithm stopped because the population of genitors was stable throughout the last 10 generation. By $\mathbf{u}_{\text {opt }}$ the best ranked individual from the last generation generated by the algorithm is denoted. The last three columns of Table 2 show the value of the flow, the cost and the resilience of the network represented by $\mathbf{u}_{\text {opt }}$. The comparison of the obtained $\mathbf{u}_{\text {opt }}$ with the results of exact calculations confirms that the proposed algorithm indeed resulted in the optimum solution for every $t=1, \ldots, 6$.

Model 2. Recall that in this model the value of the flow is maximised subject to constraints on the cost and the resilience of the network. Therefore, the problem 


\begin{tabular}{|c|c|c|c|c|c|}
\hline$t$ & $n_{\text {gen }}$ & $\mathbf{u}_{\text {opt }}$ & $\psi\left(\mathbf{u}_{\text {opt }}\right)$ & $c\left(\mathbf{u}_{\text {opt }}\right)$ & $R\left(\mathbf{u}_{\text {opt }}, t\right)$ \\
\hline \hline 1 & 15 & $(1,3,0,2,2,0,1,3,0)$ & 3 & 13.8 & 0.584 \\
\hline 2 & 19 & $(2,2,0,2,2,0,0,3,0)$ & 3 & 12.5 & 0.796 \\
\hline 3 & 20 & $(1,3,0,1,2,1,0,3,0)$ & 3 & 12.8 & 0.848 \\
\hline 4 & 19 & $(1,3,0,1,2,0,1,2,1)$ & 3 & 13.1 & 0.884 \\
\hline 5 & 18 & $(1,3,0,1,2,0,0,3,2)$ & 3 & 13.8 & 0.900 \\
\hline 6 & 19 & $(1,3,1,1,2,0,0,3,0)$ & 3 & 12.8 & 0.902 \\
\hline
\end{tabular}

Table 3: The computational results of Model 2 by using the proposed genetic algorithm.

can be written as

$$
\begin{array}{ll}
\max & \psi(\mathbf{u}) \\
\text { s.t. } & c(\mathbf{u}) \leq c_{0} \text { and } \\
& R(\mathbf{u}, t) \geq R_{0}
\end{array}
$$

We set that $c_{0}=14$ and $R_{0}$ equals $0.58,0.779,0.838,0.878,0.892,0.9$ for $t=1, \ldots, 6$ respectively. Setting the same $\lambda=400$ and $\mu=50$ and by using the same stopping criterion as in the previous model, the proposed genetic algorithm yields the results presented in Table 3 . The comparison of the obtained $\mathbf{u}_{o p t}$ with the results of exact calculations confirms that the proposed algorithm indeed resulted in the optimum solution for every $t=1, \ldots, 6$.

Model 3. Recall that in this model the resilience of the network in the time $t$ is maximised subject to constraints on the cost and the value of the flow in the network. Therefore, the problem can be written as

$$
\begin{array}{ll}
\max & R(\mathbf{u}, t) \\
\text { s.t. } & c(\mathbf{u}) \leq c_{0} \text { and } \\
& \psi(\mathbf{u}) \geq \psi_{0}
\end{array}
$$

We set that $c_{0}=18$ and $\psi_{0}=4$. Setting the same $\lambda=400$ and $\mu=50$ and using the same stopping criterion as in previous models, the proposed genetic algorithm yields the results presented in Table 4 . The comparison of the obtained $\mathbf{u}_{\text {opt }}$ with the results of exact calculations confirms that the proposed algorithm indeed resulted in the optimum solution for every $t=1, \ldots, 6$. 


\begin{tabular}{|c|c|c|c|c|c|}
\hline$t$ & $n_{\text {gen }}$ & $\mathbf{u}_{\text {opt }}$ & $\psi\left(\mathbf{u}_{\text {opt }}\right)$ & $c\left(\mathbf{u}_{\text {opt }}\right)$ & $R\left(\mathbf{u}_{\text {opt }}, t\right)$ \\
\hline \hline 1 & 21 & $(2,3,0,2,2,1,1,3,1)$ & 4 & 17.4 & 0.601 \\
\hline 2 & 18 & $(2,3,0,2,2,1,1,3,1)$ & 4 & 17.4 & 0.786 \\
\hline 3 & 23 & $(2,3,0,2,2,1,1,3,1)$ & 4 & 17.4 & 0.862 \\
\hline 4 & 17 & $(2,3,0,2,2,1,1,3,1)$ & 4 & 17.4 & 0.893 \\
\hline 5 & 23 & $(2,3,0,2,2,1,1,3,1)$ & 4 & 17.4 & 0.906 \\
\hline 6 & 19 & $(2,3,0,2,2,1,1,3,1)$ & 4 & 17.4 & 0.912 \\
\hline
\end{tabular}

Table 4: The computational results of Model 3 by using the proposed genetic algorithm.

\section{Acknowledgements}

This research is partially supported through project KK.01.1.1.02.0027, a project co-financed by the Croatian Government and the European Union through the European Regional Development Fund - the Competitiveness and Cohesion Operational Programme.

\section{References}

[1] A. Asadabadi, E. Miller-Hooks, Optimal transportation and shoreline infrastructure investment planning under a stochastic climate future, Transp. Res. Part B 100, 156-74, 2017.

[2] K. Barker, J. E. Ramirez-Marquez, C. M. Rocco, Resilience-based network component importance measures, Reliab. Eng. Syst. Saf. 117, 89-97, 2013.

[3] H. Baroud, K. Barker, J. E. Ramirez-Marquez, C. M. Rocco, Inherent costs and interdependent impacts of infrastructure network resilience, Risk. Anal. 35(4), 642-662, 2015.

[4] M. Chertoff, National infrastructure protection plan, Department of Homeland Security (DHS), Washington, DC 2009.

[5] M. Christopher, H. Peck, Building the resilient supply chain, Int. J. Logist. Manage. 15(2), 1-14, 2004. 
[6] Y. Fang, N. Pedroni, E. Zio, Optimization of cascade-resilient electrical infrastructures and its validation by power flow modeling, Risk. Anal. 35(4), 594-607, 2015 .

[7] R. Faturechi, E. Miller-Hooks, Travel time resilience of roadway networks under disaster, Transp. Res. Part B 70, 47-64, 2014.

[8] C. S. Holling, Resilience and stability of ecological systems, Annu. Rev. Ecol. Syst. 1-23, 1973.

[9] S. Hosseini, K. Barker, J. E. Ramirez-Marquez, A review of definitions and measures of system resilience, Reliab. Eng. Syst. Saf. 145, 47-61, 2016.

[10] J. A. Nachlas, Reliability engineering: probabilistic models and maintenance methods, CRC Press, 2005.

[11] T. P. Runarsson, X. Yao, Stochastic ranking for constrained evolutionary optimization, IEEE Transactions on Evolutionary Computation 4(3), 284-294, 2000 .

[12] N. Yodo, P. Wang, Resilience allocation for early stage design of complex engineered systems, J. Mech. Des. 138(9), 091402, 2016.

[13] X. Zhang, S. Mahadevan, S. Sankararaman, K. Goebel, Resilience-based network design under uncertainty, Reliab. Eng. Syst. Saf. 169, 364-79, 2018. 
